\title{
Simulation of Interference Between Earth Stations and Earth-Orbiting Satellites
}

\author{
D. F. Bishop \\ Telecommunications Systems Section
}

\begin{abstract}
It is often desirable to determine the potential for radio frequency interference between Earth stations and orbiting spacecraft. This information can be used to select frequencies for radio systems to avoid interference or it can be used to determine if coordination betwen radio systems is necessary. A model is developed that will determine the statistics of interference between Earth stations and elliptical orbiting spacecraft. The model uses orbital dynamics, detailed antenna patterns, and spectral characteristics to obtain accurate levels of interference at the victim receiver. The model is programmed into a computer simulation to obtain longterm statistics of interference. Two specific examples are shown to demonstrate the model. The first example is a simulation of interference from a fixed-satellite Earth station to an orbiting scatterometer receiver. The second example is a simulation of interference from Earth-exploration satellites to a decp-space Earth station.
\end{abstract}

\section{Theory and Models}

Figure 1 contains an illustration of the interference geometry for Earth orbiters and an Earth station. Spacecraft 1 may be transmitting or receiving. Its antenna is pointed toward an arbitrary location on Earth. The Earth station may be transmitting or receiving. Its antenna is pointed toward spacecraft 2 or toward an arbitrary point described by the Earth station antenna azimuth and elevation.

Two interference scenarios are considered. In the first scenario, the Earth station is transmitting a signal toward spacecraft 2. This signal is unintentionally received by spacecraft 1 . In the second scenario, spacecraft 1 is transmitting a signal that is unintentionally received by the Earth station.

The interference geometry shown in Fig. 1 is common to many interference scenarios that occur between two dif- ferent radio systems. The level of interference that occurs at a victim receiver depends on angles $\gamma_{g}$ and $\gamma_{r g}$ and the distance $D_{s f}$ that are shown in Fig. 1. The primary emphasis of the first part of this article is to show how to compute these parameters as a function of time. The antenna gain of spacecraft 1 in the direction of the Earth station is a function of angle $\gamma_{g}$. The path loss between spacccraft 1 and the Earth station is a function of the distance $D_{s f}$. Lastly, the antenna gain of the Earth station in the direction of spacecraft 1 is a function of angle $\gamma_{r g}$. The antenna gains and path loss are used to determine interference levels at the victim receiver as a function of time.

The interference angles and path distance in Fig. 1 may be computed with standard orbit determination methods [1]. The appendix contains detailed derivations of the angles and path distance. 
The International Telecommunication Union (ITU) antenna pattern is used to calculate the Earth station antenna gain [2].

$$
\begin{aligned}
G\left(\gamma_{r g}\right)= & G_{p}-2.5 \times 10^{-3}\left(\frac{D \gamma_{r g}}{\lambda}\right)^{2}(\mathrm{dBi}), \\
& 0 \mathrm{deg} \leq \gamma_{r g}<\phi_{m} \\
= & G_{1}, \quad \phi_{m} \leq \gamma_{r g}<\phi_{r} \\
= & 32-25 \times \log \left(\gamma_{r g}\right), \quad \phi_{r} \leq \gamma_{r g}<48 \mathrm{deg} \\
= & -10, \quad 48 \mathrm{deg} \leq \gamma_{r g}<180 \mathrm{deg}
\end{aligned}
$$

where

$$
\begin{aligned}
G_{p} & =\text { peak antenna gain, } \mathrm{dBi} \\
D & =\text { antenna diameter, } \mathrm{m} \\
\lambda & =\text { wavelength }=c / f, \mathrm{~m} \\
G_{1} & =2+15 \times \log (D / \lambda), \mathrm{dBi} \\
\phi_{m} & =20 \lambda\left(G_{p}-G_{1}\right)^{1 / 2} / D, \mathrm{deg} \\
\phi_{r} & =15.85(D / \lambda)^{-0.6}, \mathrm{deg} \\
c & =\text { speed of light }=3 \times 10^{8}, \mathrm{~m} / \mathrm{sec} \\
f & =\text { frequency, } \mathrm{Hz}
\end{aligned}
$$

This equation is valid for $D / \lambda \geq 100$. For $D / \lambda<100$, a different pattern is used.

$$
\begin{aligned}
G\left(\gamma_{r g}\right)= & G_{p}-2.5 \times 10^{-3}\left(\frac{D \gamma_{r g}}{\lambda}\right)^{2}(\mathrm{dBi}), \\
& 0 \mathrm{deg} \leq \gamma_{r g}<\phi_{m} \\
= & G_{1}, \quad \phi_{m} \leq \gamma_{r g}<\frac{100 \lambda}{D} \\
= & 52-10 \log \left(\frac{D}{\lambda}\right)-25 \times \log \left(\gamma_{r g}\right), \\
& \frac{100 \lambda}{D} \leq \gamma_{r g}<48 \mathrm{deg} \\
= & 10-10 \log \left(\frac{D}{\lambda}\right),
\end{aligned}
$$$$
48 \mathrm{deg} \leq \gamma_{r g}<180 \mathrm{deg}
$$

The path loss from spacecraft 1 to the Earth station is computed.

$$
P L=20 \times \log \left(\frac{c}{\left[4 \pi D_{s f} f\right]}\right)(\mathrm{dB})
$$

The angle $\gamma_{g}$ in Fig. 1 is used to compute the antenna gain of spacecraft 1 in the direction of the Earth station. The spacecraft gain pattern is mission dependent.

\section{Application 1: Simulation of Interference From a Fixed-Satellite Earth Station to a Scatterometer Receiver and Calculation of Scatterometer Power Flux Density}

\section{A. Introduction}

The World Administrative Radio Conference of 1992 (WARC-92) allocated the 13.75- to $14-\mathrm{GHz}$ frequency band to fixed-satellite (Earth-to-space) service on a primary basis. NASA scatterometers are permitted to use this band under the category of space research (spacecraft radiolocation) service on a secondary basis. The International Radio Consultative Committee (CCIR) formulated Task Group 7/3 (TG 7/3) to study the use of the 13.4to $14-\mathrm{GHz}$ frequency band by science services. One of the goals of TG $7 / 3$ was to determine the effect that the new allocation (to fixed-satellite service) would have on NASA scatterometers near $14 \mathrm{GHz}$. The models developed in Section I and the appendix were used to generate a sinulation of interference from a fixed-satellite Earth station to a scatterometer in Earth orbit. The scatterometer is represented by spacecraft 1 and the fixed-satellite spacecraft is represented by spacecraft 2 in Fig. 1. The fixed-satellite Earth station is represented by the Earth station in Fig. 1.

\section{B. Analysis}

The computation of the scatterometer antenna gain in the direction of the fixed-satellite Earth station depends on components of the angle $\gamma_{y}$ in Fig. 1. The scatterometer antenna coordinate and spacecraft coordinate systems are illustrated in Fig. 2. There are six antenna beams, whose positions are defined by angles $\alpha, \beta$, and $\gamma$. A vector in the antemna coordinate system can be transformed into a vector in the spacecraft coordinate system.

$$
\mathbf{x}_{\mathbf{s}}=\mathbf{A} \mathbf{x}_{\mathbf{a}}
$$

where

$$
\mathbf{A}=\left[\begin{array}{ccc}
A & D & G \\
B & E & H \\
C & F & I
\end{array}\right]
$$




$$
\begin{aligned}
& \mathrm{A}=\cos (\gamma) \cos (\alpha) \\
& \mathrm{B}=\cos (\gamma) \cos (90-\alpha) \\
& \mathrm{C}=\cos (90-\gamma) \\
& \mathrm{D}=-\cos (90-\alpha) \cos (\beta)+\cos (90-\beta) \cos (90-\gamma) \cos (\alpha) \\
& \mathrm{E}=\cos (\alpha) \cos (\beta)+\cos (90-\beta) \cos (90-\gamma) \cos (90-\alpha) \\
& \mathrm{F}=-\cos (90-\beta) \cos (\gamma) \\
& \mathrm{G}=-[\cos (90-\alpha) \cos (90-\beta)+\cos (90-\gamma) \cos (\beta) \cos (\alpha)] \\
& \mathrm{H}=\cos (\alpha) \cos (90-\beta)-\cos (90-\gamma) \cos (\beta) \cos (90-\alpha) \\
& \mathrm{I}=\cos (\beta) \cos (\gamma)
\end{aligned}
$$

A vector in the spacecraft coordinate system can be transformed into a vector in the orbit-plane coordinate system.

$$
\mathbf{x}_{\mathbf{w a}}=\mathbf{B} \mathbf{x}_{\mathbf{s}}
$$

where

$$
\begin{aligned}
& \mathbf{B}=\left[\begin{array}{ccc}
-\frac{y_{w}}{a} & 0 & -\frac{x_{w}}{a} \\
\frac{x_{w}}{a} & 0 & -\frac{y_{w}}{a} \\
0 & -1 & 0
\end{array}\right] \\
& \mathbf{a}=\text { semimajor axis (Earth radii) }
\end{aligned}
$$

A vector in the orbit-plane coordinate system can be transformed into the right ascension-declination coordinate system.

$$
\mathbf{x}_{\mathbf{a n}}=\mathbf{P} \mathbf{x}_{\mathbf{w a}}
$$

where $\mathbf{P}$ is defined in Eq. (A-3) in the appendix.

The vector (from the scatterometer spacecraft to the fixed-satellite Earth station) is transformed from the right ascension-declination coordinate system into the antennacoordinate system. This is done by converting from the right ascension-declination system to the orbit-plane system, to the spacecraft system, and to the antenna system.

$$
\mathbf{x}_{\mathbf{g f a}}=-\mathbf{A}^{T} \mathbf{B}^{T} \mathbf{P}^{T} \mathbf{x}_{\mathbf{f s}}=\left(x_{s f a}, y_{s f a}, z_{s f a}\right)^{T}
$$

These components are used to determine the off-axis angles in the narrow-beam plane and wide-beam plane of the fan beam antenna (Fig. 3). The antenna boresight points in the $\mathbf{k}$ direction.

$$
\begin{aligned}
& \cos \left(\beta_{g}\right)=\frac{z_{s f a}}{\left(x_{s f a}{ }^{2}+z_{s f a}\right)^{1 / 2}} \\
& \cos \left(\alpha_{g}\right)=\frac{z_{s f a}}{\left(y_{s f a}{ }^{2}+z_{s f a}\right)^{1 / 2}}
\end{aligned}
$$

These angles are used to compute the narrow-beam and wide-beam components of the fan beam antenna gain.

$$
\begin{aligned}
G_{1}\left(\beta_{g}\right)= & -3\left(\frac{\beta_{g}}{0.173}\right)^{2}, \quad 0 \mathrm{deg} \leq \beta_{g}<0.5 \mathrm{deg} \\
& \text { (narrow-beam component, dBi) } \\
= & -23.9-2.16 \beta_{g}, \quad 0.5 \mathrm{deg} \leq \beta_{g}<5.6 \mathrm{deg} \\
= & -48, \quad 5.6 \mathrm{deg} \leq \beta_{g}<180 \mathrm{deg} \\
G_{2}\left(\alpha_{g}\right)= & 34-3\left(\frac{\alpha_{g}}{13.9}\right)^{2}, \quad 0 \mathrm{deg} \leq \alpha_{g}<20 \mathrm{deg} \\
& (\text { wide-beam component, } \mathrm{dBi}) \\
= & 35.83-0.402 \alpha_{g}, \quad 20 \mathrm{deg} \leq \alpha_{g}<114 \mathrm{deg} \\
= & -14.5, \quad 114 \mathrm{deg} \leq \alpha_{g}<180 \mathrm{deg}
\end{aligned}
$$

The fan beam antenna gain in the direction of the fixedsatellite Earth station is the summation of the narrowbeam and wide-beam components.

$$
G_{f b}=G_{1}\left(\beta_{g}\right)+G_{2}\left(\alpha_{g}\right)
$$

where the minimum value of $G_{f b}$ is $-14 \mathrm{dBi}$. At this point, there is enough information to compute the level of interference from the fixed-satellite Earth station to the scatterometer.

$$
I=P_{T}+G\left(\gamma_{r g}\right)+S L+P L+G_{f b}
$$

where

$$
\begin{aligned}
\mathrm{I} & =\text { interference power spectral density }(\mathrm{dBW} / \mathrm{Hz}) \\
P_{T} & =\text { fixed-satellite Earth station transmitter power } \\
& =85-53.4 \mathrm{dBW}
\end{aligned}
$$




$$
\begin{aligned}
\mathrm{G}\left(\gamma_{r g}\right)= & \text { fixed-satellite Earth station antenna gain in di- } \\
& \text { rection of scatterometer [Eq. (1)] } \\
G_{p}= & 53.4 \mathrm{dBi} \\
\mathrm{D}= & 4.5 \mathrm{~m} \\
\mathrm{f}= & 13.995 \mathrm{GHz} \\
\mathrm{SL}= & 10 \times \log (1 / \mathrm{BW})(\mathrm{dBW} / \mathrm{Hz}) \\
\mathrm{BW}= & \text { bandwidth }=2 \mathrm{MHz} \\
\mathrm{PL}= & \text { path loss from fixed-satellite Earth station to } \\
& \text { scatterometer [Eq. (3)], dB } \\
G_{f b}= & \text { fan beam antenna gain in direction of fixed- } \\
& \text { satellite Earth station [Eq. (10)], dBi }
\end{aligned}
$$

The threshold level where interference is considered harmful to the scatterometer is $-207 \mathrm{dBW} / \mathrm{Hz} .{ }^{1}$ The ground track of the scatterometer is computed to provide a visual indication of where the scatterometer is interfered with. The latitude of the ground track is computed first.

$$
L_{a g t}=90-\cos ^{-1}\left(\frac{z}{a}\right)
$$

Then, the longitude is computed by

$$
\begin{aligned}
L_{o g t} & =L_{o 1}-\frac{360 t}{1436.1}, \quad y \geq 0 \\
& =-L_{o 1}-\frac{360 t}{1436.1}, \quad y<0
\end{aligned}
$$

where

$$
L_{o 1}=\cos ^{-1}\left[\frac{x}{a\left(\sin \left[90-L_{a g t}\right]\right)}\right]
$$

The longitude is transformed to obtain values between -180 and $180 \mathrm{deg}$.

\section{Statistics and Probability of Interference}

When the scatterometer is visible to the fixed-satellite Earth station, it is possible for interference to occur. The duration of these interference episodes varies with each orbit of the scatterometer. The scatterometer-simulation

${ }^{1}$ M. Spencer, "Interference Criterion for Ku-Band Scatterometer," JPL Interoffice Memorandum (internal document), Jet Propulsion Laboratory, Pasadena, California, December 8, 1992. program computes the maximum and average times of an interference episode for the complete simulation period. Then histograms of these interference episodes are generated and the distribution of interference episodes may be estimated.

The scatterometer measures wind speed over the ocean. Taking this measurement requires that three antenna beams (beams $1-3$ or beams 4-6) have no interference. The effect of interference from a fixed-satellite Earth station to the scatterometer is to create certain areas on the ocean where measurements of the wind speed cannot be taken by the scatterometer. It is possible that the scatterometer will have interference on the ascending portion of the orbit over a particular area of the Earth. When the scatterometer passes over this area on a descending portion of the orbit, it is possible that there will be no interference. Therefore, it is desirable to compute the statistics of interference for areas of the Earth.

For a simple example, the Earth is divided into areas of $100 \mathrm{~km} \times 100 \mathrm{~km}$ near the equator. To facilitate programming, this is done by dividing the Earth into areas that are 0.89833 -deg longitude in width and 0.89833 -deg latitude in height. The width of these areas is $100 \mathrm{~km}$ at the equator, tapering off to a width of $0 \mathrm{~km}$ at the north and south poles. The antenna beams are very narrow in one direction and wide in the orthogonal direction. Therefore, the beams are approximated with 31 vectors in the widebeam plane of the antenna. These vectors are spaced $1 \mathrm{deg}$ apart, yielding a total coverage of $30 \mathrm{deg}$ in the wide-beam plane. The point of intersection of an antenna vector with the Earth is determined.

Figure 4 shows the geometry. The unit vector $\mathbf{v} /|\mathbf{v}|$ is converted from antenna coordinates to the right ascension declination coordinate system. Then angle $\alpha_{v}$ can be calculated. The magnitude of $\mathbf{v}$ and the vector $\mathbf{R}$ can be determined by

$$
\begin{aligned}
-\mathbf{x} \cdot \frac{\mathbf{v}}{|\mathbf{v}|} & =a\left(\cos \left[\alpha_{v}\right]\right) \\
|\mathbf{R}|^{2} & =a^{2}+|\mathbf{v}|^{2}-2 a|\mathbf{v}| \cos \left(\alpha_{v}\right) \\
\mathbf{R} & =\mathbf{x}+\mathbf{v}
\end{aligned}
$$

where

$$
\frac{\mathbf{v}}{|\mathbf{v}|}=\left(v_{x}, v_{y}, v_{z}\right)^{T}
$$


This vector location is converted to latitude and longitude [Eqs. (12) and (13)]. Then, the area to which this point belongs is determined.

Wind-speed measurements on the right side of the scatterometer are taken by combining signals from beams $1-3$ and, on the left side, by combining signals from beams 46. An area on the right side is considered to be covered if a vector from beams 1-3 intersects the area on the same orbit and the interference is below the receiver interference threshold for beams 1-3. Likewise, an area on the left side is considered to be covered if a vector from beams 4-6 intersects the area on the same orbit and the interference is below the receiver interference threshold for beams 4-6. The probability of interference is estimated as

$$
p=\frac{n}{d}
$$

where

$$
\begin{aligned}
& p=\text { estimate of probability of area interference } \\
& n=\text { the number of times that an area is covered and } \\
& \text { interfered with } \\
& d=\text { the number of times that an area is covered }
\end{aligned}
$$

where the definitions of "covered" and "interfered with" are provided in the paragraph above.

\section{Power Flux Density of the Scatterometer}

Compatibility between terrestrial and spacecraft radio systems is often determined from the power flux density of the spacecraft on the surface of the Earth. Therefore, the power flux density of the scatterometer is computed. Figure 5 contains an illustration of the geometry.

$$
P_{f d}=P_{t}+G_{2}\left(\alpha_{g}\right)+F S S
$$

where

$$
\begin{aligned}
P_{f d}= & \text { power flux density of scatterometer at surface } \\
& \text { of the Earth, } \mathrm{dBW} / \mathrm{m}^{2} \\
P_{t}= & \text { scatterometer transmit power, dBW } \\
G_{2}\left(\alpha_{g}\right)= & \text { scatterometer transmit antenna gain as a } \\
& \text { function of angle }\left(\alpha_{g}\right) \text { in the wide-beam plane } \\
& \text { (Fig. } 3), \mathrm{dBi} \\
F S S= & \text { free space spreading loss, } \mathrm{dB} / \mathrm{m}^{2} \\
= & 10 \times \log \left(1 /\left[4 \pi\left(6378 \times 10^{3} D_{s e}\right)^{2}\right]\right)
\end{aligned}
$$

The direction of the antenna gain vector is computed in the antenna coordinate system (Fig. 3).

$$
\mathbf{x}_{\mathbf{v a}}=\left(0, \cos \left[90-\alpha_{g}\right], \cos \left[\alpha_{g}\right]\right)^{T}
$$

This vector is transformed into the right ascensiondeclination coordinate system with Eqs. (4-6).

$$
\mathbf{x}_{\mathbf{v r}}=\mathbf{P B A} \mathbf{A x}_{\mathbf{v a}}=\left(x_{v r}, y_{v r}, z_{v r}\right)^{T}
$$

Then, the angle $\left(\alpha_{s e}\right)$ is computed by

$$
\mathbf{x} \bullet \mathbf{x}_{\mathbf{v r}}=-a\left[\cos \left(\alpha_{s e}\right)\right]
$$

The distance between the scatterometer and the point of intersection with the Earth can be computed by

$$
D_{s e}=a\left[\cos \left(\alpha_{s e}\right)\right]-\left[\left(a\left[\cos \left(\alpha_{s e}\right)\right]\right)^{2}-\left(a^{2}-1\right)\right]^{1 / 2}
$$

The vector to the point of intersection of the antenna gain vector and the Earth is computed by

$$
\mathbf{x}_{\mathbf{c r}}=\mathbf{x}+D_{s e} \mathbf{x}_{\mathbf{v r}}
$$

The latitude and longitude of this point, $L_{a e}$, and $L_{o e}$, are computed with Eqs. (12) and (13).

It is desirable to know the power flux density as a function of elevation angle on the Earth. Therefore, the elevation of the vector $-\mathbf{x}_{\mathbf{v r}}$ is computed.

$$
\begin{aligned}
E_{l}= & \sin ^{-1}\left(-\mathrm{x}_{\mathrm{vr}} \cos \left[L_{a e}\right] \cos \left[L_{o e}\right]-y_{v r} \cos \left[L_{a e}\right]\right. \\
& \left.\times \cos \left[90-L_{o e}\right]-z_{v r} \cos \left[90-L_{a e}\right]\right)
\end{aligned}
$$

\section{E. Simulation}

Table 1 shows the results of a sample simulation. Interference is considered harmful if it exceeds the threshold shown on the table. The fixed-satellite Earth station and geostationary satellite are arbitrarily located. Interference is computed at 1 -sec intervals. The estimated probability of interference is the number of interference minutes divided by simulation minutes. The scatterometer orbital period is approximately $100 \mathrm{~min}$. Because the position of the orbit changes relative to the fixed-satellite Earth 
station, some orbits have no interference and other orbits have episodes of interference for varying amounts of time. The maximum and mean of these interference episodes are provided.

\section{Application 2: Simulation of Interference from an Earth-Exploration Satellite to a Deep-Space Earth Station}

\section{A. Introduction}

The Deep Space Network (DSN) uses the 8400- to 8450$\mathrm{MHz}$ band for space-to-Earth transmissions. Earth-station receivers are protected by interference criteria that have been negotiated in international forums. Other radio services that use this band are aware of the interference criteria and limit their transmissions accordingly. However, radio services that transmit in adjacent bands may not be fully aware of their emissions in the DSN band. If these out-of-band emissions are strong enough, they can disrupt DSN communications.

\section{B. Power Spectral Density of Adjacent Band Interferers}

The power spectral density from an Earth-orbiting spacecraft is computed with the following equation:

$$
P_{S D}=P_{T}+S D(f)+G_{T}+P L_{1}+G_{R}
$$

$$
\begin{aligned}
\text { where } & \\
P_{S D}= & \text { power spectral density of interfering space- } \\
& \text { craft at a deep-space Earth station receiver, } \\
& \mathrm{dBW} / \mathrm{Hz} \\
P_{T}= & \text { spacecraft transmitter power, } \mathrm{dBW} \\
S D(f)= & \text { spectral density of spacecraft transmitter } \\
& \text { (Table } 2), \mathrm{dB} / \mathrm{Hz} \\
G_{T}= & \text { peak transmit antenna gain, } \mathrm{dBi} \\
P L_{1}= & \text { path loss, } \mathrm{dB} \\
= & 20 \times \log \left[c /\left(4 \pi A_{m} f\right)\right] \\
c= & \text { speed of light }=3 \times 10^{5} \mathrm{~km} / \mathrm{s} \\
A_{m}= & \text { minimum orbit altitude, } \mathrm{km} \\
f= & \text { frequency, } \mathrm{Hz} \\
G_{R}= & \text { DSN receive antenna gain }=74 \mathrm{dBi}
\end{aligned}
$$

The spectral density is computed from the equations shown in Table $2[3] .^{2}$

In particular, the Earth Observing System (EOS) program is planning the launch of several spacecraft [4] that will have spectral emissions in the 8400 - to $8450-\mathrm{MHz}$ band. Using Eq. (23), the emission of an EOS spacecraft is determined to exceed the DSN interference criterion of $-220.9 \mathrm{dBW} / \mathrm{Hz}$ by about $45 \mathrm{~dB}$ in the 8400 - to $8450-\mathrm{MHz}$ band. Equation (23) produces a worst-case power spectral density at the deep-space Earth station because it uses peak antenna gains and the minimum orbit altitude to compute the path loss. Statistics of the interference power spectral density are provided next.

\section{Simulation of Interference to a Deep-Space Earth Station from Low Earth-Orbiting Spacecraft in an Adjacent Band}

It is useful to know the amount of time that the power spectral density from an adjacent-band spacecraft exceeds the interference criteria of the deep-space Earth station. Figure 6 shows a plot of satellite visibility to an Earth station versus orbit altitude. It is assumed that the satellite passes directly over the Earth station with a circular orbit. The geometry is illustrated in Fig. 7. Visibility time is computed as

$$
T_{v i s}=\frac{3600 T_{o} \theta}{\pi}(\mathrm{sec})
$$

where

$$
\begin{aligned}
T_{o} & =\text { orbital period, } \mathrm{hr}[5] \\
& =2 \pi(A+R)^{3 / 2} / \mu^{1 / 2} \\
A & =\text { orbit altitude, } \mathrm{km} \\
R & =\text { Earth radius }=6378 \mathrm{~km} \\
\mu & =\text { Earth gravitation, mass product } \\
& =5.17 \times 10^{12} \mathrm{~km}^{3} / \mathrm{hr}^{2} \\
\theta & =\text { central angle, } \mathrm{rad} \\
& =\cos ^{-1}(\mathrm{R} /[\mathrm{R}+\mathrm{A}])
\end{aligned}
$$

If the satellite has sufficient power, it can produce lineof-sight interference to the Earth station during its orbit visibility time. This can be a significant amount of time even for a low-altitude spacecraft. Interference times for

\footnotetext{
${ }^{2}$ B. Tunstall, "Transmit Filtering for EOS Direct Access System Downlink," Memorandum to J. Scott (internal document), Goddard Space Flight Center, Greenbelt, Maryland, July 10, 1992.
} 
actual spacecraft systems depend on transmitter power, orbits, antenna gains, etc. Figure 1 can be used to illustrate the interference geometry. The EOS spacecraft is represented with spacecraft 1 . The deep-space Earth station points at a specified azimuth and elevation. The EOS spacecraft is in a circular orbit around the Earth with its antenna pointing toward the center of the Earth. The angle $\gamma_{r g}$ between the boresight of the deep-space Earth station and the vector to the EOS spacecraft is computed with Eqs. (A-15) and (A-17) in the appendix. The deepspace Earth station $(70-\mathrm{m})$ antenna gain in the direction of the EOS spacecraft is computed with Eq. (1).

$$
\begin{aligned}
G\left(\gamma_{r g}\right)= & 74-0.0025\left(1960 \gamma_{r g}\right)^{2}(\mathrm{dBi}) \\
& 0 \mathrm{deg} \leq \gamma_{r g}<0.0485 \mathrm{deg} \\
= & 51.4, \quad 0.0485 \mathrm{deg} \leq \gamma_{r g}<0.168 \mathrm{deg} \\
= & 32-25 \log \left(\gamma_{r g}\right), \quad 0.168 \mathrm{deg} \leq \gamma_{r g}<48 \mathrm{deg} \\
= & -10, \quad 48 \mathrm{deg} \leq \gamma_{r g} \leq 180 \mathrm{deg}
\end{aligned}
$$

The angle $\gamma_{g}$ between the antenna boresight of the EOS spacecraft and the vector to the deep-space Earth station is computed with Eqs. (A-19) and (A-20) in the appendix. The EOS spacecraft antenna gain in the direction of the deep-space Earth station is computed. The model is a set of straight line segments that approximates the actual pattern. ${ }^{3}$

$$
\begin{aligned}
G_{e}\left(\gamma_{g}\right) & =-3.5+\frac{2.5 \gamma_{g}}{3}(\mathrm{dBi}), \quad 0 \mathrm{deg} \leq \gamma_{g}<3 \mathrm{deg} \\
& =0.091-\frac{4 \gamma_{g}}{11}, \quad 3 \mathrm{deg} \leq \gamma_{g}<14 \mathrm{deg} \\
& =-8.5+\frac{14 \gamma_{g}}{56}, \quad 14 \mathrm{deg} \leq \gamma_{g}<70 \mathrm{deg} \\
& =84.25-\frac{21.5 \gamma_{g}}{20}, \quad 70 \mathrm{deg} \leq \gamma_{g}<90 \mathrm{deg} \\
& =-14.5, \quad 90 \mathrm{deg} \leq \gamma_{g} \leq 180 \mathrm{deg}
\end{aligned}
$$

The interference power spectral density level at the deepspace Earth station receiver is computed by

$$
I_{o}=S D(f)+P_{e}+G_{e}\left(\gamma_{g}\right)+P L+G\left(\gamma_{r g}\right)
$$

\footnotetext{
3 J. Hart, "Interference Analysis for EOS AM Direct Access System," Interoffice Memorandum (internal document), Stanford Telecom, Seabrook, Maryland, July 7, 1992.
}

where $P_{e}=$ EOS transmitter power $=11.8 \mathrm{dBW}$.

The first simulation on Table 3 is for one EOS spacecraft transmitting in the direct playback mode (QPSK, 150 MSPS per channel). Statistics of the interference are provided. The second simulation in Table 3 is for five different spacecraft. The antenna pattern for the last four spacecraft is the same as for the EOS antenna [Eq. (26)] except for additive constants to provide the correct peak antenna gain for each spacecraft. Each interference event is composed of a number of interference samples that occur at 0.5 -sec intervals. Samples are plotted on Fig. 8 for the second simulation in Table 3 . At each sample interval, the amount of interference exceeding the $-220.9 \mathrm{dBW} / \mathrm{Hz}$ criterion [6] is plotted. Some events have only one sample ( $0.5 \mathrm{sec}$ of interference) and some have up to 20 samples (10 sec of interference). The simulation ran on a Sun workstation.

The antenna pattern of the deep-space Earth station provided in Eq. (25) was developed a number of years ago for generalized Earth stations. More accurate gain data have been made available for the 70-m antennas of the DSN. ${ }^{4}$ These data have been fitted with a number of equations.

$$
\begin{aligned}
G_{m}\left(\gamma_{r g}\right)= & 74.15-0.0025\left(2400 \gamma_{r g}\right)^{2}(\mathrm{dBi}), \\
& 0 \mathrm{deg} \leq \gamma_{r g}<0.0376 \mathrm{deg}(\mathrm{dBi}) \\
= & 53.7 \\
& 0.0376 \mathrm{deg} \leq \gamma_{r g}<0.04 \mathrm{deg} \\
= & 57.4-0.025\left(1350\left[\gamma_{r g}-0.049\right]\right)^{2}, \\
& 0.04 \mathrm{deg} \leq \gamma_{r g}<0.0626 \mathrm{deg} \\
= & 49, \\
& 0.0626 \mathrm{deg} \leq \gamma_{r g}<0.0905 \mathrm{deg} \\
= & 25-23 \log \left(\gamma_{r g}\right), \\
& 0.0905 \mathrm{deg} \leq \gamma_{r g}<33.2 \mathrm{deg} \\
= & -10, \\
& 33.2 \mathrm{deg} \leq \gamma_{r g}<180 \mathrm{deg}
\end{aligned}
$$

\footnotetext{
4 D. Bathker, personal communication, DSN International Frequency Maragement Program, TDA Planning Section, Jet Propulsion Laboratory, Pasadena, California, October 1993.
} 
Figure 9 contains plots of antenna data, curve fits from Eq. (28), and the ITU pattern [2]. Equation (28) fits the data quite well for this application. The ITU model overestimates the gain data in every region except that from 0.04 to $0.06 \mathrm{deg}$. It is expected that the interference from lowEarth-orbit spacecraft would be reduced when the DSN Earth station antenna is modeled with Eq. (28).

The computer simulation was conducted with $G_{m}\left(\gamma_{r g}\right)$ replacing $G\left(\gamma_{r g}\right)$ in Eq. (27). In the first simulation, one EOS spacecraft transmitted in the direct playback mode. In the second simulation, five different spacecraft were transmitting. The interference percentage is one-fourth of that in Table 3 with the new antenna model for one EOS spacecraft. For five spacecraft, the interference percentage is approximately one-fourth of that in Table 3 with the new antenna model.

All of the simulations use an elevation of $5 \mathrm{deg}$ and an azimuth of $100 \mathrm{deg}$ for the deep-space Earth station antenna. When larger elevation angles are used, the interference percentages are decreased. This is because the spacecraft spends less time within the same Earth station antenna beamwidth at higher elevations. The interference levels are computed at 0.5 -sec intervals in all simulations.

\section{Summary and Conclusions}

A model is developed that will determine the statistics of interference between Earth stations and elliptical orbiting spacecraft. Detailed models of the geometry of Earth orbiters and Earth stations are shown. It is possible to determine antenna gains and path distances quite accurately with these models. Interference levels at the victim receiver are determined by antenna gains, path distances, and radio system characteristics. The models may be used to predict interference in a wide variety of scenarios that involves Earth stations and Earth orbiters. Two examples that make use of these models are shown.
The first example is a simulation of interference from a fixed-satellite Earth station to an orbiting scatterometer receiver. Table 1 contains a summary of a simulation that was performed. Models were developed to estimate the probability that certain areas of the Earth would not have accurate measurements due to interference. Also, the power flux density on Earth of the scatterometer signal as a function of elevation angle is determined. Results from the simulation showed that the scatterometer should use a different frequency to avoid interference from several fixed-satellite Earth stations. Simulation results compared favorably with other simulation programs. ${ }^{5}$

The second example is a simulation of interference from an Earth-exploration satellite to a deep-space Earth station. Worst-case levels of power spectral density from low Earth orbiters at the station are computed. These levels exceed the interference criteria of the deep-space Earth station in the $8400-$ to $8450-\mathrm{MHz}$ band. A simulation of interference from low Earth orbiters to a deep-space Earth station is conducted. This simulation computes the path loss and off-axis antenna gains as a function of orbital position of the low Earth orbiter. It can be used to predict the statistics of interference to the deep-space Earth station. Table 3 contains a summary of two different simulations that were performed. Results from the simulation showed that excessive coordination could be avoided if Earth-exploration satellites reduced their emissions by $20 \mathrm{~dB}$ in the $8400-$ to $8450-\mathrm{MHz}$ band. The interference statistics are in close agreement with other simulations. ${ }^{6}$

The simulations are programmed in Fortran and run on a Sun workstation. The author may be contacted for instructions on the operation of the simulation programs.

\footnotetext{
${ }^{5}$ J. Hart, personal communication, Stanford Telecom, Seabrook, Maryland, A pril 1993.

${ }^{6}$ J. Hart, "Compliance of EOS Direct Access System with DSN Protection Criteria," Interoffice Memorandum (intemal document), Stanford Telecom, Seabrook, Maryland, May 17, 1993.
}

\section{Acknowledgments}

The author gratefully acknowledges the help of Dan Bathker, Benito G.-Luaces, J. Hart (Stanford Telecom), Mike Spencer, and Carroll Winn in the development and testing of the models and simulations. Charles Ruggier is acknowledged for review of the manuscript. 
Table 1. Example of an interference simulation from a fixed-satellite Earth station to an orbiting scatterometer recelver.

\begin{tabular}{lc}
\hline \multicolumn{1}{c}{ Parameter } & Value \\
\hline Scatterometer interference threshold, dBW/Hz & -207 \\
Scatterometer altitude, km & 797 \\
Fixed-satellite Earth station & $85-\mathrm{dBW}$ \\
& $4.5-\mathrm{m}$ antenna \\
& $34 \mathrm{deg} \mathrm{N}, 128 \mathrm{deg}$ W \\
Geostationary satellite & $196 \mathrm{deg} \mathrm{W}$ \\
Simulation minutes & 10,000 \\
Number of interference computations per minute & 60 \\
Antenna beam element & 1 \\
Number of interference minutes & 2.067 \\
Estimated probability of interference & $2.067 \times 10^{-4}$ \\
Maximum period of an interference episode, min & 0.567 \\
Mean period of an interference episode, min & 0.129 \\
\hline
\end{tabular}

Table 2. Spectral density equations.

\begin{tabular}{|c|c|}
\hline Modulation & Spectral density (baseband) \\
\hline Quadriphase-shift keying & $(2 / S R) \times[\sin (2 \pi f / S R)]^{2} /(2 \pi f / S R)^{2}$ \\
\hline Unbalanced quadriphase-shift keying & $\begin{array}{c}\left(r_{I} / S R_{I}\right) \times\left[\sin \left(\pi f / S R_{I}\right)\right]^{2} /\left(\pi f / S R_{I}\right)^{2} \\
+\left(r_{Q} / S R_{Q}\right) \times\left[\sin \left(\pi f / S R_{Q}\right)\right]^{2} /\left(\pi f / S R_{Q}\right)^{2}\end{array}$ \\
\hline Phase-shift keying & $(1 / S R) \times[\sin (\pi f / S R)]^{2} /(\pi f / S R)^{2}$ \\
\hline Minimum-shift keying & {$\left[16 /\left(\pi^{2} S R\right)\right] \times[\cos (2 \pi f / S R)]^{2} /\left(1-16 f^{2} / S R^{2}\right)^{2}$} \\
\hline \multicolumn{2}{|c|}{$\begin{array}{l}S R=\text { total output symbol rate } \\
f \quad=\text { frequency } \\
r_{I}=\text { ratio of power in I channel to total power } \\
S R_{I}=\text { symbol rate of I channel } \\
r_{Q}=\text { ratio of power in } Q \text { channel to total power } \\
S R_{Q}=\text { symbol rate of } Q \text { channel }\end{array}$} \\
\hline $\begin{array}{l}\text { Aeplace } f \text { with } f-f_{c} \text { (where } f_{c} \text { is } \mathrm{t} \\
\text { density at the center frequency. }\end{array}$ & ter frequency) to obtain the spectral \\
\hline
\end{tabular}


Table 3. Simulation results comparing one EOS spacecraft to five other spacecraft-EOS (DP), SPOT-4, IRS-1B, POEM 1, and RADARSAT-1.

\begin{tabular}{lcc}
\hline \multicolumn{1}{c}{ Parameter } & EOS spacecraft & Five spacecraft \\
\hline $\begin{array}{l}\text { Duration of simulation, min } \\
\text { Interference events }\end{array}$ & $525,600(1$ year $)$ & 525,600 (1 year) \\
Interference percentage & $87.89 \times 10^{-4}$ & 128 \\
Interference duration, sec & 3.5 & $2.39 \times 10^{-3}$ \\
$\quad$ Shortest & 10 & 0.5 \\
Longest & 7.58 & 10 \\
Average & & 5.90 \\
Time between interference events, days & 1.99 & 0.08 \\
Shortest & 13.44 & 12.83 \\
Longest & 10.11 & 2.87 \\
Average & & \\
\hline
\end{tabular}
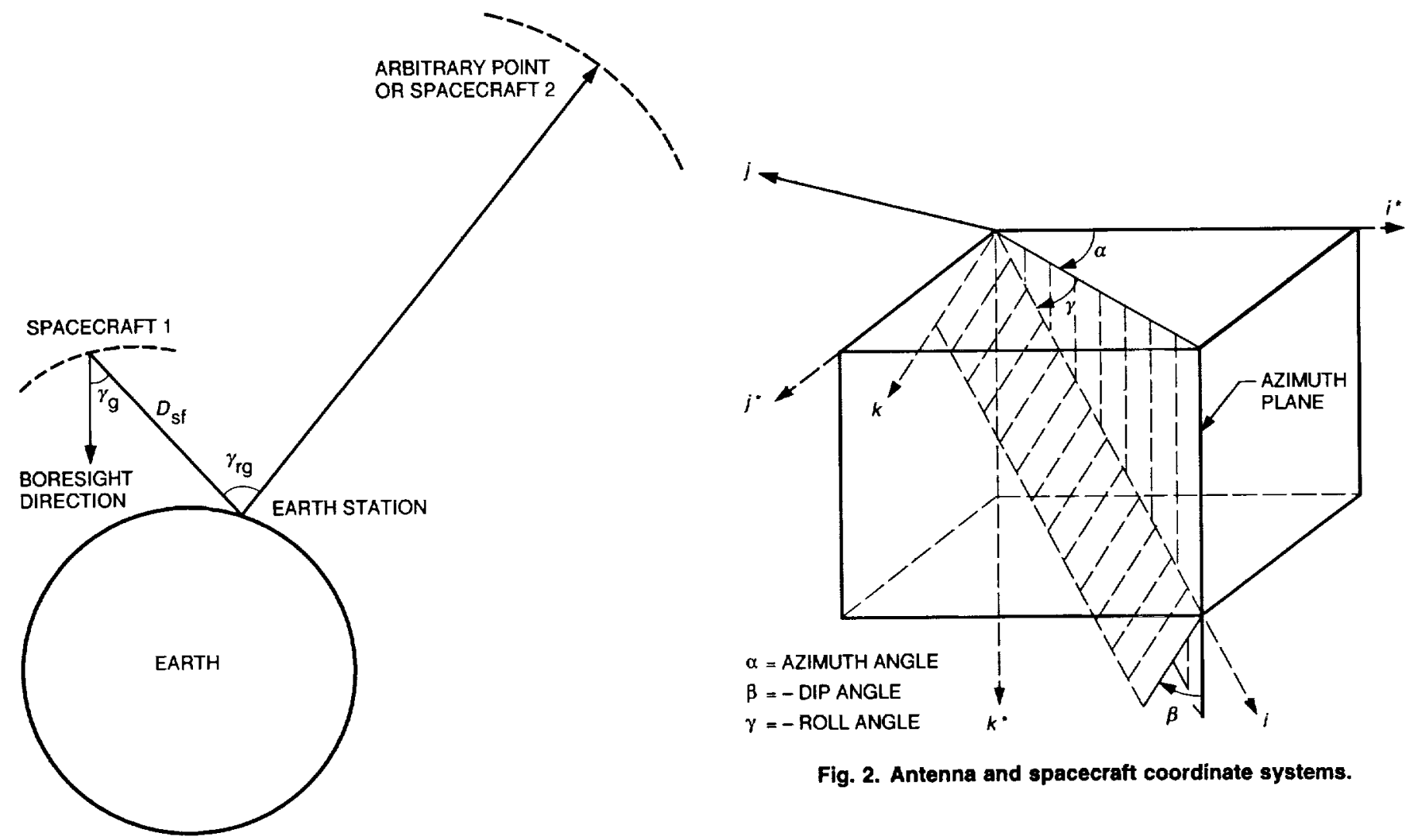

Fig. 2. Antenna and spacecraft coordinate systems.

Fig. 1. Interference geometry for Earth orbiters and an Earth station. 


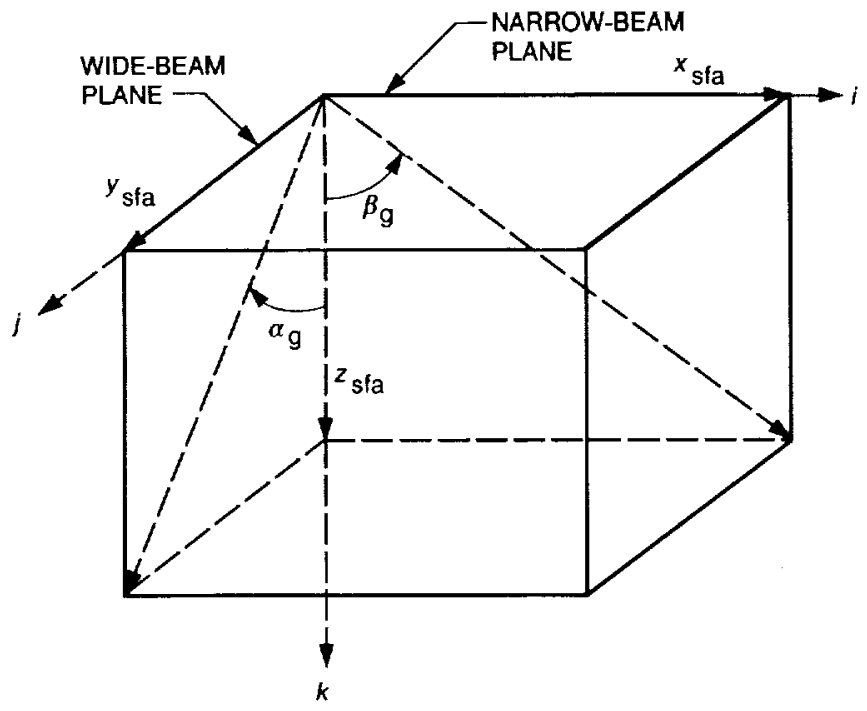

Fig. 3. Off-axis angles in the wide-beam and narrow-beam planes.

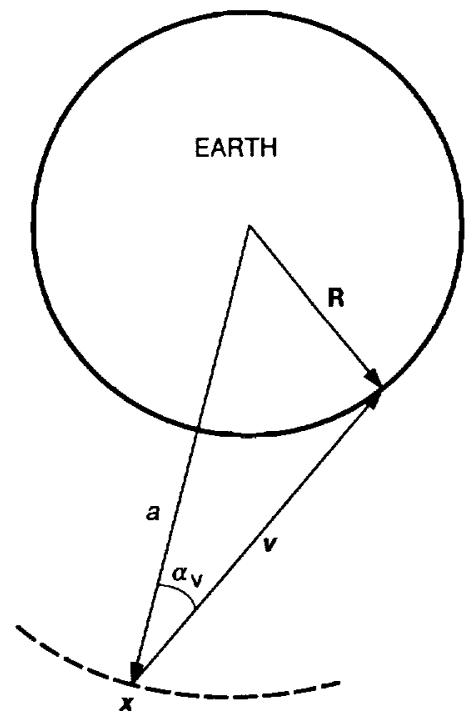

SCATTEROMETER

Fig. 4. Intersection of antenna beam with Earth.

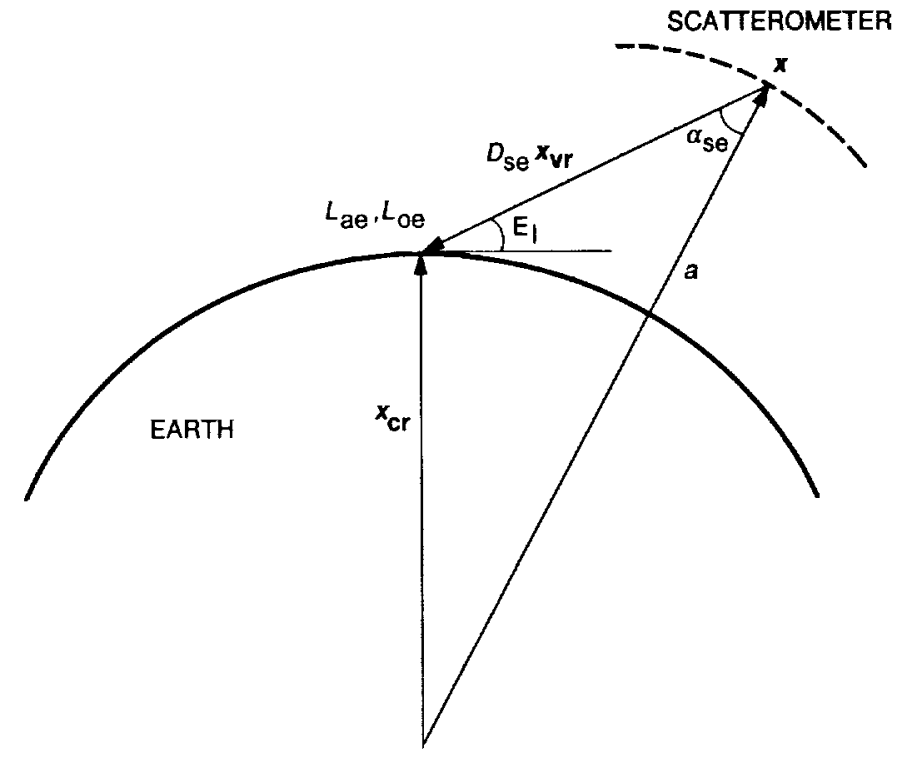

Fig. 5. Geometry for scatterometer power flux density calculation.

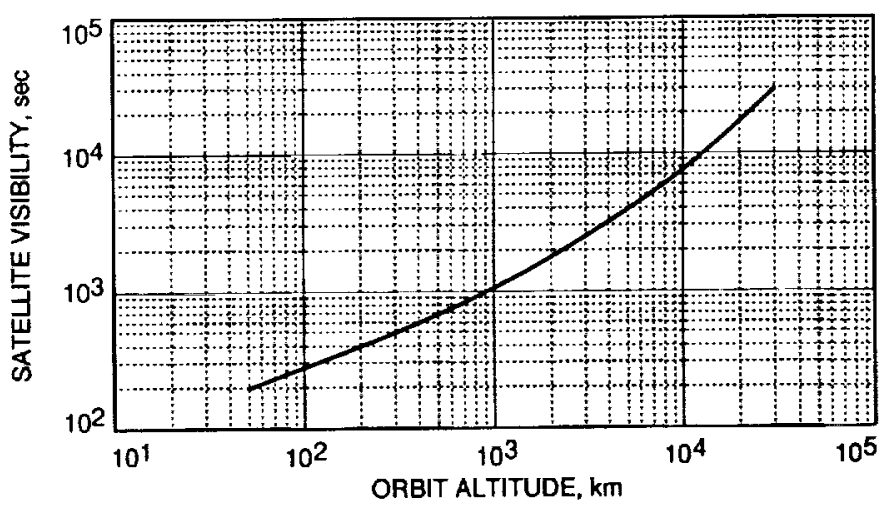

Fig. 6. Satellite visibility to an Earth station versus orbit altitude for circular satellite orbits. 


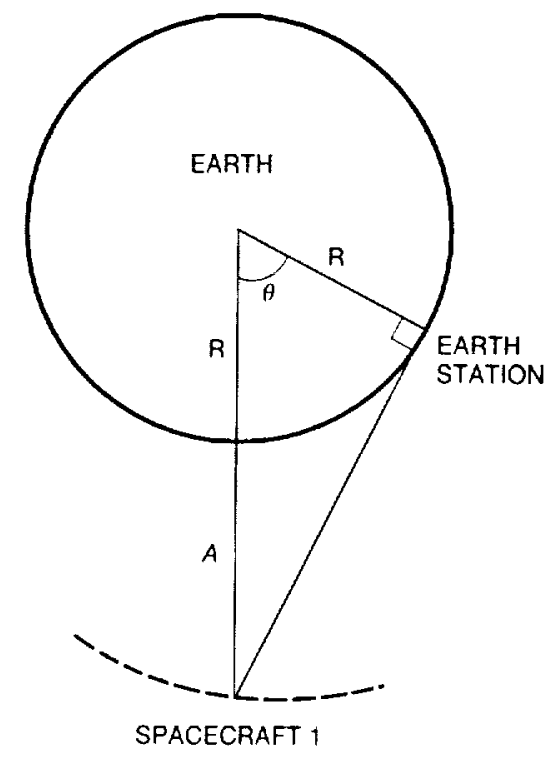

Fig. 7. Geometry for spacecraft visibility time calculation.

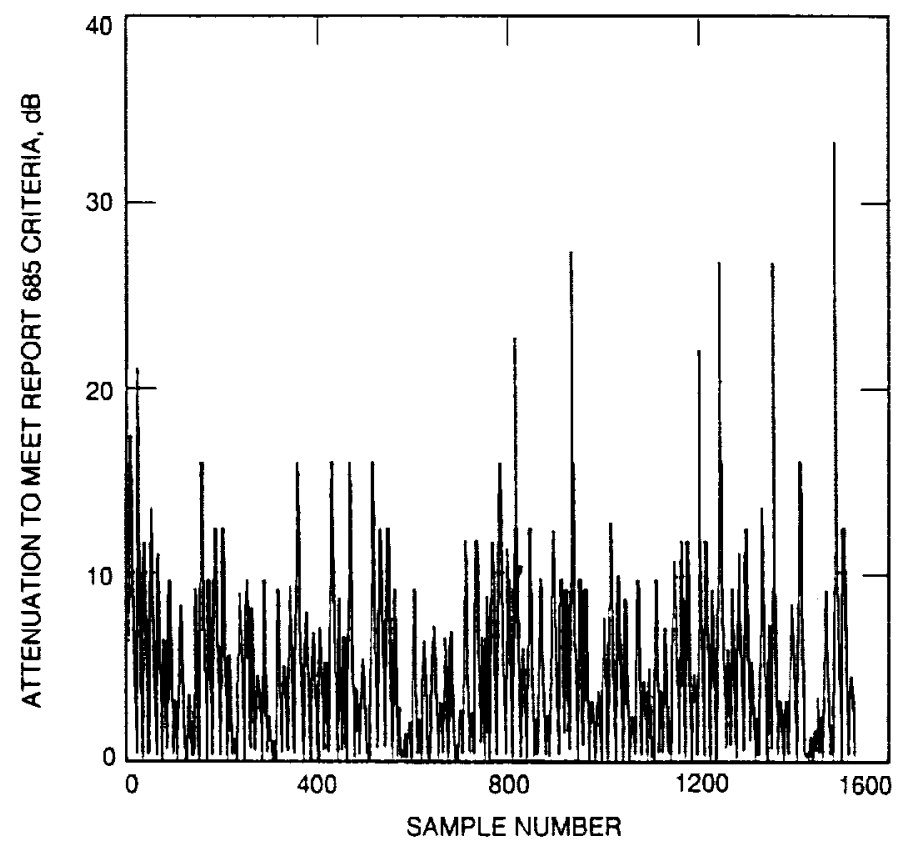

Fig. 8. Interference samples exceeding Report 685 for five other spacecraft: EOS (DP), SPOT-4, IRS-1B, POEM 1, and RADARSAT- 1 .

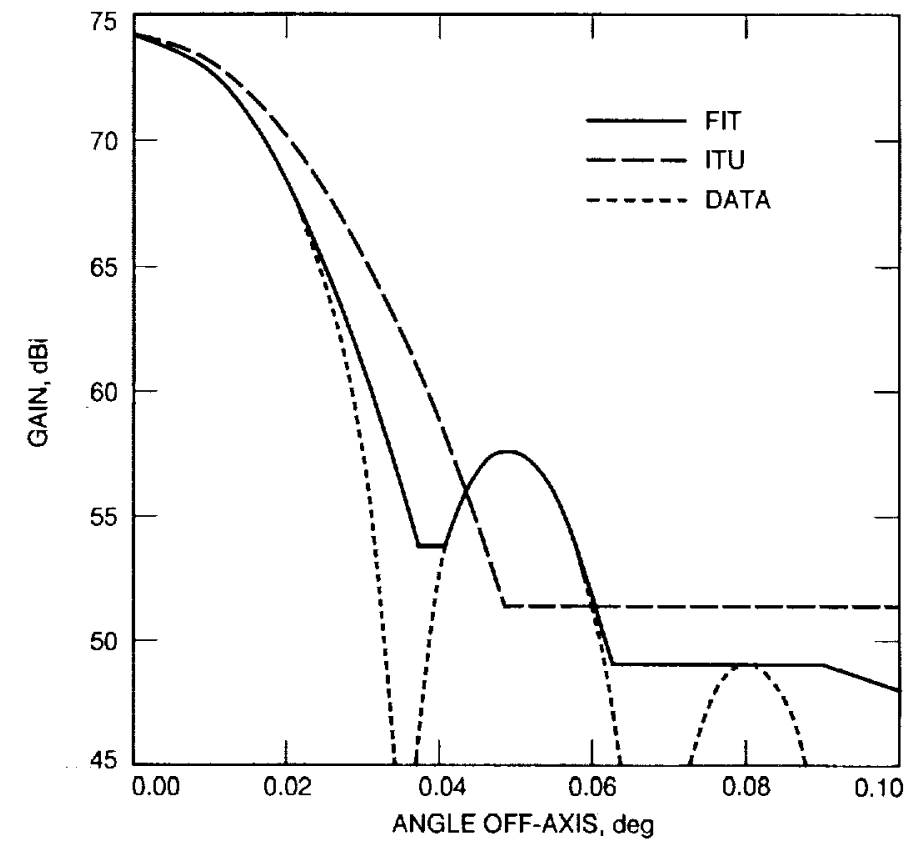

Fig. 9. Gain pattern for DSN 70-m antenna. 


\section{Appendix}

\section{Interference Geometry and Orbital Dynamics Models}

The spacecraft orbit plane is illustrated in Fig. A-1. The position of spacecraft 1 in its orbit plane is computed first.

$$
\mathbf{x}_{\mathbf{w}}=\left(a[\cos (E)]-a e, a\left[1-e^{2}\right]^{1 / 2}[\sin (E)], 0\right)^{T}
$$

where

$$
\begin{aligned}
a & =\text { semimajor axis, Earth radii } \\
E & =\text { eccentric anomaly, rad } \\
M & =E-e(\sin [E])=\text { mean anomaly, rad } \\
& =\mathrm{nt} \\
e & =\text { orbit eccentricity } \\
n & =0.07437 / a^{3 / 2}, \mathrm{rad} / \mathrm{min} \\
t & =\text { time, } \min
\end{aligned}
$$

Newton's iteration [7] may be used to solve for the eccentric anomaly, as follows:

$$
\begin{aligned}
E_{0} & =M+\frac{e(\sin [M])}{1-\sin [M+e]+\sin [M]} \\
M_{k} & =E_{k}-e\left(\sin \left[E_{k}\right]\right) \\
E_{k+1} & =E_{k}+\frac{M-M_{k}}{1-e\left[\cos \left(E_{k}\right)\right]}
\end{aligned}
$$

where

$$
k=0,1,2, \ldots
$$

The position of spacecraft 1 in the orbit plane is converted to its position in the right ascension-declination coordinate system.

$$
\mathbf{x}=\mathbf{P x}_{\mathbf{w}}=(x, y, z)^{T}
$$

where

$$
\mathbf{P}=\left[\begin{array}{lll}
P_{x} & Q_{x} & W_{x} \\
P_{y} & Q_{y} & W_{y} \\
P_{z} & Q_{z} & W_{z}
\end{array}\right]
$$

$$
\begin{aligned}
P_{x} & =\cos (\omega) \cos (\Omega)-\sin (\omega) \sin (\Omega) \cos (i) \\
P_{y} & =\cos (\omega) \sin (\Omega)+\sin (\omega) \cos (\Omega) \cos (i) \\
P_{z} & =\sin (\omega) \sin (i) \\
Q_{x} & =-\sin (\omega) \cos (\Omega)-\cos (\omega) \sin (\Omega) \cos (i) \\
Q_{y} & =-\sin (\omega) \sin (\Omega)+\cos (\omega) \cos (\Omega) \cos (i) \\
Q_{z} & =\cos (\omega) \sin (i) \\
W_{x} & =\sin (\Omega) \sin (i) \\
W_{y} & =-\cos (\Omega) \sin (i) \\
W_{z} & =\cos (i) \\
\omega & =\text { argument of perigee } \\
\Omega & =\text { longitude of the ascending node } \\
i & =\text { orbital inclination }
\end{aligned}
$$

The location of the Earth station is determined in the right ascension-declination coordinate system.

$$
\begin{aligned}
\mathbf{x}_{\mathbf{B}} & =(\sin [\Theta] \cos [\theta], \sin [\Theta] \sin [\theta], \cos [\Theta])^{T} \\
& =\left(x_{g}, y_{g}, z_{g}\right)^{T}
\end{aligned}
$$

where

$\Theta=90-l_{a}$

$\theta=l_{0}+k+360 t / 1436.1$

$k=$ an arbitrary constant used to rotate the Earth station relative to the orbit plane

$l_{a}=$ Earth station latitude

$l_{o}=$ Earth station longitude

The Earth station may be pointed at another satellite (spacecraft 2) or its boresight direction may be described with azimuth and elevation notation. If it is pointed at spacecraft 2, that satellite's position may be described with Eqs. (A-1) to (A-3). 


$$
\mathbf{x}_{\mathbf{2}}=\left(x_{2}, y_{2}, z_{2}\right)^{T}
$$

A special case exists when spacecraft 2 is in a geostationary orbit. It may be assumed that it is located in the equatorial plane. The location is determined in the right ascension-declination coordinate system.

$$
\mathbf{x}_{\mathbf{2}}=\mathbf{x}_{\mathbf{g g}}=6.6257\left(\sin \left[\Theta_{g}\right] \cos \left[\theta_{g}\right], \sin \left[\Theta_{g}\right] \sin \left[\theta_{g}\right], 0\right)^{T}
$$

where

$$
\begin{aligned}
\Theta_{g} & =90 \\
\theta_{g} & =\text { geostationary longitude }+k+360 t / 1436.1
\end{aligned}
$$

If the Earth station is pointed at a specific elevation and azimuth, its boresight direction may be converted from azimuth-elevation coordinates to Earth-centered coordinates as

$$
\mathbf{x}_{\mathbf{e c}}=\mathbf{C} \mathbf{x}_{\mathbf{a e}}=\left(x_{e c}, y_{e c}, z_{e c}\right)^{T}
$$

where

$$
\begin{aligned}
\mathbf{C}=\left[\begin{array}{lll}
C_{1} & C_{2} & C_{3} \\
C_{4} & C_{5} & C_{6} \\
C_{7} & C_{8} & C_{9}
\end{array}\right] \\
C_{1}=\cos \left(l_{o}\right) \cos \left(90-l_{a}\right) \\
C_{2}=-\cos \left(90-l_{o}\right) \\
C_{3}=\cos \left(l_{a}\right) \cos \left(l_{o}\right) \\
C_{4}=\cos \left(90-l_{o}\right) \cos \left(90-l_{a}\right) \\
C_{5}=\cos \left(l_{o}\right) \\
C_{6}=\cos \left(l_{a}\right) \cos \left(90-l_{o}\right) \\
C_{7}=-\cos \left(l_{a}\right) \\
C_{8}=0 \\
C_{9}=\cos \left(90-l_{a}\right) \\
\mathbf{x}_{\mathbf{a e}}=(-\cos [e l] \cos [a z], \cos [e l] \sin [a z], \sin [e l])^{T} \\
\text { el }=\text { elevation of Earth station antenna } \\
\text { az }=\text { azimuth of Earth station antenna }
\end{aligned}
$$

These coordinates are converted to spherical coordinates, as follows:

$$
\begin{aligned}
\Theta_{b} & =\cos ^{-1}\left(\frac{z_{e c}}{r_{e c}}\right)(\mathrm{deg}) \\
\theta_{b} & =\cos ^{-1}\left(\frac{x_{e c}}{r_{x y}}\right)+k+\frac{360 t}{1436.1}, y_{e c} \geq 0(\mathrm{deg}) \\
& =360-\cos ^{-1}\left(\frac{x_{e c}}{r_{x y}}\right)+k+\frac{360 t}{1436.1}, y_{e c}<0
\end{aligned}
$$

where

$$
\begin{aligned}
& r_{e c}=\left(x_{e c}^{2}+y_{e c}^{2}+z_{e c}^{2}\right)^{1 / 2} \\
& r_{x y}=\left(x_{e c}^{2}+y_{e c}^{2}\right)^{1 / 2}
\end{aligned}
$$

Finally, these coordinates are converted to the right ascension-declination coordinate system by

$$
\mathbf{x}_{\text {bor }}=r_{e c}\left(\sin \left[\Theta_{b}\right] \cos \left[\theta_{b}\right], \sin \left[\Theta_{b}\right] \sin \left[\theta_{b}\right], \cos \left[\Theta_{b}\right]\right)^{T}
$$

The line-of-sight visibility of the Earth station to spacecraft 1 is determined. Figure A-2 illustrates the central angle between the two. The central angle between spacecraft 1 and the Earth station is computed as

$$
\mathbf{x} \bullet \mathbf{x}_{\mathbf{g}}=|\mathbf{x}| \cos (\gamma)
$$

where $\gamma=$ central angle between spacecraft 1 and the Earth station.

Figure A-2 also illustrates the limit of visibility. The constraint is that the line between spacecraft 1 and the Earth station is tangent to the Earth. Central angles that are less than or equal to this angle indicate that spacecraft 1 is visible to the Earth station.

$$
\gamma_{v}=\cos ^{-1}\left(\frac{1}{\left|\mathbf{x}_{\mathbf{v}}\right|}\right)
$$

The visibility condition is stated as

$$
\gamma \leq \gamma_{v}
$$


Interference between spacecraft 1 and the Earth station can occur only if they are visible. If spacecraft 1 is visible, additional computations are necessary. The angle $\gamma_{r g}$ on Fig. 1 is used to compute the antenna gain of the Earth station in the direction of spacecraft 1 . The vector from the Earth station to spacecraft 2 is computed as

$$
\mathbf{x}_{\mathbf{f g}}=\mathbf{x}_{2}-\mathbf{x}_{\mathrm{g}}
$$

A special case occurs when spacecraft 2 is a geostationary satellite, as follows:

$$
\mathbf{x}_{2}=\mathbf{x}_{\mathrm{gg}}
$$

If the pointing of the Earth station antenna is described in terms of azimuth and elevation, then

$$
\mathbf{x}_{\mathbf{f g}}=\mathbf{x}_{\text {bor }}
$$

The vector from the Earth station to spacecraft 1 is computed as

$$
\mathbf{x}_{\mathbf{f s}}=\mathbf{x}-\mathbf{x}_{\mathbf{g}}
$$

The angle between these two vectors [Eqs. (A-13) and (A-16)] can be used to determine the antenna gain of the Earth station in the direction of spacecraft 1 .

$$
\mathbf{x}_{\mathbf{f g}} \bullet \mathbf{x}_{\mathbf{f s}}=\left|\mathbf{x}_{\mathrm{fg}}\right|\left|\mathbf{x}_{\mathbf{f s}}\right| \cos \left(\gamma_{r g}\right)
$$

The distance between the Earth station and spacecraft 1 is computed as

$$
D_{s f}=6378\left|\mathbf{x}_{\mathbf{g}}-\mathbf{x}\right|(k m)
$$

The angle between the antenna boresight of spacecraft 1 and the direction to the Earth station is computed as

$$
\mathbf{x}_{\mathbf{b} \mathbf{1}} \bullet\left(-\mathbf{x}_{\mathbf{f s}}\right)=\left|\mathbf{x}_{\mathbf{b} \mathbf{1}}\right|\left|\mathbf{x}_{\mathbf{f s}}\right| \cos \left(\gamma_{g}\right)
$$

where $\mathbf{x}_{\mathbf{b} \mathbf{1}}=$ antenna boresight vector of spacecraft 1.

A special case occurs when the antenna of spacecraft 1 points toward the center of the Earth.

$$
\mathbf{x}_{\mathbf{b} \mathbf{1}}=-\mathbf{x}
$$




\section{References}

[1] P. R. Escobal, Methods of Orbit Determination, New York: Wiley, 1976.

[2] International Telecommunication Union Radio Regulations, ISBN 92-61-01221-3, vol. 2, 1982.

[3] S. Pasupathy, "Minimum Shift Keying: A Spectrally Efficient Modulation," IEEE Communications Society Magazine, vol. 17, no. 4, pp. 14-22, July 1979.

[4] Goddard Space Flight Center, Mission Requirements and Data Systems Support Forecast, Greenbelt, Maryland, pp. 18-23, April-May 1993.

[5] Reference Data for Radio Engineers, New York: Howard W. Sams \& Co., Inc., 1982.

[6] CCIR, "Protection Criteria and Sharing Considerations Relating to Deep-Space Research," Reports of the CCIR, annex to vol. II, Space Research and Radioastronomy Services, CCIR Report 685-3, Geneva, Switzerland, pp. 281-302, 1990.

[7] R. H. Battin, Introduction to the Mathematics and Methods of Astrodynamics, New York: American Institute of Aeronautics and Astronautics, 1987. 